\title{
HIGHER PICARD VARIETIES AND THE HEIGHT PAIRING
}

\author{
KLAUS KÜNNEMANN
}

\begin{abstract}
Let $X$ be a smooth projective variety which is defined over a number field. Beilinson and Bloch have defined under suitable asssumptions height pairings between Chow groups of homologically trivial cycles on X. Beilinson has also formulated a hard Lefschetz and a Hodge index conjecture for these Chow groups. We show that the restriction of the height pairing to cycles algebraically equivalent to zero can be computed via Abel-Jacobi maps in terms of the Néron-Tate height pairing on the higher Picard varieties of $X$. This description is used in the case where $X$ is an abelian variety to prove a consequence of Beilinson conjectures. Namely, we prove a hard Lefschetz and a Hodge index theorem for the groups of cycles algebraically equivalent to zero modulo incidence equivalence.
\end{abstract}

\section{INTRODUCTION}

Let $X_{K}$ be a smooth projective variety of dimension $d$ defined over the number field $K$. Beilinson and Bloch have defined under suitable assumptions height pairings

$$
<.,>_{X_{K}}: C H^{p}\left(X_{K}\right)_{\mathbf{Q}}^{0} \times C H^{d+1-p}\left(X_{K}\right)_{\mathbf{Q}}^{0} \longrightarrow \mathbf{R}
$$

between the Chow groups of homologically trivial cycles ([Be], $[\mathrm{Bl}])$. Beilinson has also conjectured analogues of Grothendieck's standard conjectures for the Chow groups of homologically trivial cycles. Namely, these groups should satisfy a hard Lefschetz theorem and an analogue of the Hodge index theorem should hold for the pairing (1). We denote by $A^{p}\left(X_{K}\right)_{\mathbf{Q}}$ the subgroup of $C H^{p}\left(X_{K}\right)_{\mathbf{Q}}^{0}$ consisting of cycles which become algebraically equivalent to zero over an algebraic closure $\bar{K}$ of $K$. The restricted pairing

$$
<., .>_{X_{K}}: A^{p}\left(X_{K}\right)_{\mathbf{Q}} \times A^{d+1-p}\left(X_{K}\right)_{\mathbf{Q}} \longrightarrow \mathbf{R}
$$

is well defined under the assumption that $X_{K}$ has a regular model which is projective and flat over the ring of integers in $K$. The higher Picard varieties $\operatorname{Pic}^{p}\left(X_{\bar{K}}\right)$ are abelian varieties which parametrize cycles algebraically equivalent to zero modulo incidence equivalence on $X_{\bar{K}}$. Using the Abel-Jacobi map

$$
\theta^{p}: A^{p}\left(X_{K}\right) \longrightarrow \operatorname{Pic}^{p}\left(X_{\bar{K}}\right)(\bar{K}),
$$

the pairing (2) can be described in terms of the Néron-Tate pairing on the higher Picard varieties. A similiar description in terms of the Néron-Tate pairing on the

This article is copyrighted to the Johns Hopkins University Press, which will publish it in the American Journal of Mathematics. 
algebraic part of the intermediate Jacobians was indicated by Beilinson in [Be], 4.0.8. We denote by $B^{p}\left(X_{K}\right)_{\mathbf{Q}}$ the quotient of $A^{p}\left(X_{K}\right)_{\mathbf{Q}}$ by the subgroup of cycles which become incidence equivalent to zero over $\bar{K}$. Conjecturally, $B^{p}\left(X_{K}\right)_{\mathbf{Q}}$ equals $A^{p}\left(X_{K}\right)_{\mathbf{Q}}$.

Let $A_{K}$ be an abelian variety which has a regular model which is projective and flat over the ring of integers in $K$. Beilinson's conjectures predict a hard Lefschetz and a Hodge index theorem for the groups $A^{p}\left(A_{K}\right)_{\mathbf{Q}}$. The aim of this note is to show that these theorems hold for the subquotients $B^{p}\left(A_{K}\right)_{\mathbf{Q}}$ of $C H^{p}\left(A_{K}\right)_{\mathbf{Q}}^{0}$. The hard Lefschetz theorem for $B^{p}\left(A_{K}\right)_{\mathbf{Q}}$ is derived from a hard Lefschetz theorem for Chow motives of abelian varieties. One only has to show that $n$-multiplication on $A_{K}$ induces $\left(n^{2 p-1}\right)$-multiplication on $\operatorname{Pic}^{p}\left(A_{\bar{K}}\right)$. We use the description of (2) in terms of the Néron-Tate pairing on the higher Picard varieties to prove the Hodge index theorem. The crucial point is to show that the Fourier transform - an algebraic correspondence which induces the Hodge-*-operator in singular cohomology induces a polarization of $\operatorname{Pic}^{p}\left(A_{\bar{K}}\right)$. The higher Picard varieties are closely related to intermediate Jacobians. The positivity of the Hodge---operator implies that the Fourier transform defines a polarization of Weil's intermediate Jacobians. In this way, we derive our Hodge index theorem for the groups $B^{p}\left(A_{K}\right)_{\mathbf{Q}}$ from the positivity of the Néron-Tate pairing and the positivity of the Hodge-*-operator.

\section{Preliminaries}

For an abelian group $G$, we set $G_{\mathbf{Q}}=G \otimes_{\mathbf{Z}} \mathbf{Q}$. Let $X$ be a scheme. We denote by $C H_{p}(X)$ (resp. $C H^{p}(X)$ ) the Chow group of dimension (resp. codimension) $p$ cycles on $X$. Let $k$ be a field. A variety over $k$ is a separated scheme of finite type over $k$ which is geometrically integral. We denote the category of smooth projective varieties over $k$ by $\mathcal{V}_{k}$. Let $X$ and $Y$ be varieties in $\mathcal{V}_{k}$. A correspondence $\alpha \in C H^{\bullet}\left(X \times_{k} Y\right)$ induces a map

$$
\alpha_{C H}: C H^{\bullet}(X) \longrightarrow C H^{\bullet}(Y), x \mapsto p_{Y *}\left(p_{X}^{*}(x) . \alpha\right)
$$

where $p_{X}$ and $p_{Y}$ are the obvious projection maps. The group $A^{p}(X)$ is by definition the subgroup of $C H^{p}(X)$ consisting of cycle classes which become algebraically equivalent to zero over an algebraic closure $\bar{k}$ of $k$ ([Fu], Ch. 10.3).

Let $A$ be an abelian variety defined over an algebraic closure $\overline{\mathbf{Q}}$ of $\mathbf{Q}$. We denote the dual abelian variety by $A^{\vee}$. The Néron-Tate pairing

$$
(., .)_{A}: A(\overline{\mathbf{Q}}) \times A^{\vee}(\overline{\mathbf{Q}}) \longrightarrow \mathbf{R}
$$

is defined in [Né]. It yields a positive definite bilinear form $(x, y) \mapsto(x, \lambda(y))_{A}$ on $A(\overline{\mathbf{Q}})_{\mathbf{Q}}$ for every polarization $\lambda: A \rightarrow A^{\vee}$ of $A$. Let $f: A \rightarrow B$ be a homomorphism of abelian varieties. We denote by $f^{\vee}: B^{\vee} \rightarrow A^{\vee}$ the dual morphism which satisfies

$$
(f(x), y)_{B}=\left(x, f^{\vee}(y)\right)_{A} \quad \text { for } x \in A(\overline{\mathbf{Q}}), y \in B^{\vee}(\overline{\mathbf{Q}}) .
$$




\section{Arithmetic intersection theory}

We recall some basic facts from arithmetic intersection theory as developped by Gillet and Soulé in [GS1], [GS2]. The arithmetic intersection product is defined for regular arithmetic varieties. However regular schemes are not stable under products and base extensions. This yields problems when we deal with correspondences. We use the cap products defined in [GS2] to avoid them.

An arithmetic variety is by definition a scheme $X$ which is projective and flat over $\operatorname{Spec}(\mathbf{Z})$, and has a smooth generic fiber $X_{\mathbf{Q}}$. Let $X$ be an arithmetic variety. Complex conjugation defines an involution $F_{\infty}: X(\mathbf{C}) \rightarrow X(\mathbf{C})$ on the complex manifold $X(\mathbf{C})$. We denote by $A^{p, p}\left(X_{\mathbf{R}}\right)$ the space of real valued differential forms $\alpha$ on $X(\mathbf{C})$ which are of type $(p, p)$ and satisfy $F_{\infty}^{*}(\alpha)=(-1)^{p} \alpha$. The space $D_{p, p}\left(X_{\mathbf{R}}\right)$ is the space of real currents $X(\mathbf{C})$ which is the topological dual of $A^{p, p}\left(X_{\mathbf{R}}\right)$ with respect to the Schwartz topology. Let $A_{p, p}\left(X_{\mathbf{R}}\right) \subset D_{p, p}\left(X_{\mathbf{R}}\right)$ be the subspace of currents represented by smooth forms.

Let $Z_{p}(X)$ be the group of dimension $p$ cycles on $X$. A cycle $Z=\sum_{i} n_{i}\left[Z_{i}\right] \in$ $Z_{p}(X)$ defines a current $\delta_{Z} \in D_{p-1, p-1}\left(X_{\mathbf{R}}\right)$ by the formula $\delta_{Z}(\eta)=\sum_{i} n_{i} \int_{Z_{i}(\mathbf{C})^{n s}} \eta$. The subgroup of $Z_{p}(X) \oplus D_{p, p}\left(X_{\mathbf{R}}\right)$ consisting of all pairs $(Z, g)$ where $g$ is a Green current for $Z$, i.e. $d d^{c} g+\delta_{Z}=\omega$ for some smooth form $\omega \in A_{p-1, p-1}\left(X_{\mathbf{R}}\right)$, is by definition the group $\hat{Z}_{p}(X)$ of arithmetic cycles of dimension $p$. The arithmetic

Chow homology group $\widehat{C H}_{p}(X)$ is the quotient of $\hat{Z}_{p}(X)$ by the subgroup generated by elements $(0, \partial u+\bar{\partial} v)$ and $\widehat{\operatorname{div}}(f)$, where $W$ is an integral subscheme of dimension $p+1, f \in k(W)^{*}$, and $\widehat{\operatorname{div}}(f)$ is the arithmetic cycle $\left(\operatorname{div}(f),-\left[\log |f|^{2}\right]\right)$. We set

$$
\widetilde{A}_{p, p}\left(X_{\mathbf{R}}\right)=\frac{A_{p, p}\left(X_{\mathbf{R}}\right)}{\operatorname{Im}(\partial)+\operatorname{Im}(\bar{\partial})} \quad \text { and } \quad H_{p, p}\left(X_{\mathbf{R}}\right)=\operatorname{Ker}\left(\left.d d^{c}\right|_{\widetilde{A}_{p, p}\left(X_{\mathbf{R}}\right)}\right) .
$$

The natural maps

$$
\begin{aligned}
\omega: \widehat{C H}_{p}(X) \longrightarrow A_{p-1, p-1}\left(X_{\mathbf{R}}\right) \quad, \quad \alpha=[(Z, g)] \mapsto \omega(\alpha)=d d^{c} g+\delta_{Z} \\
a: \widetilde{A}_{p, p}\left(X_{\mathbf{R}}\right) \longrightarrow \widehat{C H}_{p}(X) \quad, \quad[\eta] \mapsto a(\eta)=[(0, \eta)], \\
\zeta: \widehat{C H}_{p}(X) \longrightarrow C H_{p}(X) \quad, \quad[(Z, g)] \mapsto[Z] \\
\mathrm{cl}: \widehat{C H}_{p}(X) \longrightarrow H_{p, p}\left(X_{\mathbf{R}}\right) \quad, \quad \alpha \mapsto \operatorname{cl}(\alpha)=[\omega(\alpha)]
\end{aligned}
$$

fit into an exact sequence

$$
\widetilde{A}_{p, p}\left(X_{\mathbf{R}}\right) \stackrel{a}{\longrightarrow} \widehat{C H}_{p}(X) \stackrel{\zeta}{\longrightarrow} C H_{p}(X) \longrightarrow 0 .
$$

The arithmetic Chow homology groups are covariant for proper maps between arithmetic varieties, which restrict to smooth maps in the generic fiber. They are contravariant (with a shift of the degree) for local complete intersection (l.c.i.) morphisms and for flat maps, which are generically smooth.

We obtain the arithmetic Chow groups $\widehat{C H}^{p}(X)$ if we grade by codimension instead of dimension. They have properties analogous to those stated above for the 
Chow homology groups. We have $\widehat{C H}^{p}(X)=\widehat{C H}_{d+1-p}(X)$ if $X$ is regular and equidimensional of absolute dimension $d+1$.

Let $Y$ be a regular arithmetic variety. The arithmetic intersection product, constructed in [GS1] and denoted by '.', makes $\widehat{C H}^{\bullet}(Y)_{\mathbf{Q}}=\oplus_{p} \widehat{C H}^{p}(Y)_{\mathbf{Q}}$ into a graded commutative ring. Given a morphism $f: X \longrightarrow Y$ of arithmetic varieties, with $Y$ regular, there is also a cap product

$$
\widehat{C H}^{p}(Y) \otimes \widehat{C H}_{q}(X) \longrightarrow \widehat{C H}_{q-p}(X)_{\mathbf{Q}}, y \otimes x \mapsto y \cdot f
$$

which defines on $\widehat{C H} \cdot(X)_{\mathbf{Q}}$ the structure of a graded $\widehat{C H}^{\bullet}(Y)_{\mathbf{Q}}$ module. The following properties of the cap product are easily derived from its definition and Theorem 3 in [GS2], 2.3. Given morphisms $g: X \rightarrow U$ and $h: W \rightarrow X$ between arithmetic varieties with $U$ regular and $h$ smooth in the generic fibre, $x \in \widehat{C H} \cdot(X)$, $u \in \widehat{C H}^{\bullet}(U), w \in \widehat{C H} \bullet(W), y \in \widehat{C H}^{\bullet}(Y), \eta \in \widetilde{A}^{\bullet}\left(Y_{\mathbf{R}}\right)$, and $\phi \in \widetilde{A}_{\bullet}\left(X_{\mathbf{R}}\right)$, we have

$$
\begin{aligned}
u \cdot g(y \cdot f x) & =y \cdot f(u \cdot g), \\
y \cdot f\left(h_{*}(w)\right) & =h_{*}(y \cdot f h w), \\
\omega(y \cdot f x) & =f^{*} \omega(y) \wedge \omega(x), \\
a(\eta) \cdot f & =a\left(f^{*} \eta \wedge \omega(x)\right), \\
y \cdot f a(\phi) & =a\left(f^{*} \omega(y) \wedge \phi\right) .
\end{aligned}
$$

The construction of the cap product in [GS2], 2.3 also yields a product

$$
C H^{p}(Y) \otimes C H_{q}(X) \longrightarrow C H_{q-p}(X)_{\mathbf{Q}}, y \otimes x \mapsto y \cdot f x
$$

for the usual Chow groups which is compatible with the map $\zeta$.

\section{Arithmetic InterseCtion PAIRINGS}

Let $X$ be an arithmetic variety. We define

$$
C H(X)^{0}=\operatorname{Ker}\left(\operatorname{cl}: C H(X) \longrightarrow H\left(X_{\mathbf{R}}\right)\right) .
$$

Let $h$ be a Kähler metric on $X(\mathbf{C})$ which is invariant under $F_{\infty}$. The pair $\bar{X}=(X, h)$ is called an Arakelov variety. The Arakelov Chow group $C H^{p}(\bar{X})\left(\right.$ resp. $C H_{p}(\bar{X})$ ) of $\bar{X}$ is by definition the inverse image of the subgroup of harmonic forms in $A^{p, p}\left(X_{\mathbf{R}}\right)$ (resp. $\left.A_{p-1, p-1}\left(X_{\mathbf{R}}\right)\right)$ under the map $\omega$. It fits into an exact sequence

$$
H^{p-1, p-1}\left(X_{\mathbf{R}}\right) \stackrel{a}{\longrightarrow} C H^{p}(\bar{X}) \stackrel{\zeta}{\longrightarrow} C H^{p}(X) \longrightarrow 0 .
$$

It is important to note that every lift of an element in $C H^{p}(X)^{0}$ to $C H^{p}(\bar{X})$ is contained in the kernel of $\omega$.

Let $f: X \longrightarrow Y$ be a morphism of arithmetic varieties, with $Y$ regular. The arithmetic degree map

$$
\widehat{\operatorname{deg}}: \widehat{C H}_{0}(X) \longrightarrow \mathbf{R}
$$


is defined in [GS1], 3.4. Given $F_{\infty}$-invariant Kähler metrics on $X(\mathbf{C})$ and $Y(\mathbf{C})$, we get a pairing

$$
C H^{p}(\bar{Y}) \times C H_{p}(\bar{X}) \longrightarrow \widehat{C H}_{0}(X) \stackrel{\widehat{\operatorname{deg}}}{\longrightarrow} \mathbf{R} .
$$

Observing (9) and (10), we obtain a well defined pairing

$$
<.,{ }_{f}: C H^{p}(Y)^{0} \times C H_{p}(X)^{0} \longrightarrow \mathbf{R} .
$$

which does not depend on the choice of the Kähler metrics on $X(\mathbf{C})$ and $Y(\mathbf{C})$. If $X=Y$ is regular and equidimensional of absolute dimension $d+1$, then $(12)$ becomes the pairing

$$
<.,>_{X}: C H^{p}(X)^{0} \times C H^{d+1-p}(X)^{0} \longrightarrow \mathbf{R} .
$$

which was already introduced in [GS1], 4.2.10, 4.3.1.

The pairing (13) behaves well under the action of correspondences. Let $K$ be a number field, $\mathcal{O}_{K}$ its ring of integers, and $S=\operatorname{Spec}\left(\mathcal{O}_{K}\right)$. Let $f: X \rightarrow Y$ and $g: W \rightarrow V$ be morphisms between arithmetic varieties with $Y, V$ regular. We assume furthermore that $X$ and $W$ are flat projective $S$-schemes. Given a correspondence $\alpha \in C H_{\bullet}\left(X \times_{S} W\right)_{\mathbf{Q}}$, the transposed correspondence ${ }^{t} \alpha$ and $\alpha$ itself induce maps

$$
\begin{aligned}
{ }^{t} \alpha_{C H}: C H^{\bullet}(V)_{\mathbf{Q}} \longrightarrow C H_{\bullet}(X)_{\mathbf{Q}} \quad, \quad v \mapsto p_{X *}\left(v \cdot p_{V} \alpha\right), \\
\alpha_{C H}: C H^{\bullet}(Y)_{\mathbf{Q}} \longrightarrow C H_{\bullet}(W)_{\mathbf{Q}} \quad, \quad y \mapsto p_{W *}\left(y \cdot p_{Y} \alpha\right),
\end{aligned}
$$

where $p_{T}, T \in\{X, Y, W, V\}$, denotes the canonical map from $X \times{ }_{S} W$ to $T$.

\section{Lemma 4.1.}

$$
<y,\left({ }^{t} \alpha\right)_{C H}(v)>_{f}=<v, \alpha_{C H}(y)>_{g} \quad \text { for } y \in C H^{\bullet}(Y)_{\mathbf{Q}}^{0}, v \in C H^{\bullet}(V)_{\mathbf{Q}}^{0} .
$$

Proof. We choose Kähler metrics on the complex manifolds associated with $X, Y$, $W, V$ and $X \times{ }_{S} W$. We use a bar to denote a preimage of an element from a Chow group in the corresponding Arakelov Chow group.

$$
\begin{aligned}
& <y,\left({ }^{t} \alpha\right)_{C H}(v)>_{f}=\widehat{\operatorname{deg}}\left(\bar{y} \cdot f \overline{p_{X *}\left(v_{p_{V}} \alpha\right)}\right) \quad \text { (by definition) } \\
& =\widehat{\operatorname{deg}}\left(\bar{y} \cdot f p_{X *} \overline{\left(v_{\cdot p_{V}} \alpha\right)}\right) \quad \text { (by (4) and (10)) } \\
& =\widehat{\operatorname{deg}}\left(p_{X *}\left(\bar{y} \cdot p_{Y} \overline{\left(v \cdot p_{V} \alpha\right)}\right) \quad(\text { by }(7))\right. \\
& =\widehat{\operatorname{deg}}\left(\bar{y} \cdot p_{Y}\left(\bar{v}_{p_{V}} \bar{\alpha}\right)\right) \quad \text { (by (4) and (10)) }
\end{aligned}
$$

Our claim follows with (6) by symmetry. 


\section{The HEIGHT PAIRING}

Let $K$ be a number field and $X_{K}$ a variety in $\mathcal{V}_{K}$ of dimension $d$. Beilinson and Bloch have defined under suitable assumptions height pairings

$$
<.,>_{X_{K}}: C H^{p}\left(X_{K}\right)_{\mathbf{Q}}^{0} \times C H^{d+1-p}(X)_{\mathbf{Q}}^{0} \longrightarrow \mathbf{R} .
$$

where $C H^{p}\left(X_{K}\right)^{0}=\operatorname{Ker}\left(\mathrm{cl}: C H^{p}\left(X_{K}\right) \longrightarrow H^{p, p}\left(X_{\mathbf{R}}\right)\right)$. In the following, we recall a definition of Beilinson's pairing which is based on Gillet and Soulé's arithmetic intersection theory ([Be], 3.1, [GS1], 4.3.1, 5.1.4).

A model for $X_{K}$ is by definition an arithmetic variety $X$ which is projective and flat over $S=\operatorname{Spec}\left(\mathcal{O}_{K}\right)$ together with an isomorphism identifying $X_{K}$ with the fiber of $X$ over $\operatorname{Spec}(K)$. We have to make the following assumption in order to apply the theory of the preceding section.

Assumption 5.1. The variety $X_{K}$ has a regular model $X$ over $S$.

We define $C H_{\text {fin }}^{p}(X)_{\mathbf{Q}}=\operatorname{Ker}\left(C H^{p}(X)_{\mathbf{Q}} \longrightarrow C H^{p}\left(X_{K}\right)_{\mathbf{Q}}\right)$ and denote by $C H_{\text {fin }}^{p}(X)_{\mathbf{Q}}^{\perp}$ the orthogonal complement to $C H_{\text {fin }}^{p}(X)_{\mathbf{Q}}$ with respect to the pairing (14). Let $C H^{p}\left(X_{K}\right)_{\mathbf{Q}}^{00}$ be the image of the canonical map

$$
\xi: C H_{\text {fin }}^{d+1-p}(X)_{\mathbf{Q}}^{\perp} \longrightarrow C H^{p}\left(X_{K}\right)_{\mathbf{Q}}^{0} .
$$

We obtain a pairing

$$
<.,>_{X_{K}}: C H^{p}\left(X_{K}\right)_{\mathbf{Q}}^{00} \times C H^{d+1-p}\left(X_{K}\right)_{\mathbf{Q}}^{00} \longrightarrow \mathbf{R}
$$

which is defined as follows. Given elements $x=\xi\left(x^{\prime}\right)\left(x^{\prime} \in C H_{\mathrm{fin}}^{d+1-p}(X)_{\mathbf{Q}}^{\perp}\right)$ and $y=\xi\left(y^{\prime}\right)\left(y^{\prime} \in C H_{\text {fin }}^{p}(X) \stackrel{\perp}{\mathbf{Q}}\right)$, we set

$$
<x, y>_{X_{K}}=<x^{\prime}, y^{\prime}>_{X}
$$

which does not depend on the choices of $x^{\prime}$ and $y^{\prime}$ (but might a priori depend on the choice of $X$ ). This pairing yields the desired height pairing (15) under the assumption

$$
C H^{p}\left(X_{K}\right)_{\mathbf{Q}}^{0}=C H^{p}\left(X_{K}\right)_{\mathbf{Q}}^{00} \text { for all } p \geq 0 \text {. }
$$

This equality is conjectured by Bloch and Beilinson. It holds for divisors and points (compare 8.1) and in the case where $X$ has a smooth model over $S$ ([Be], 2.2.6).

Let $Y_{K}$ be another variety in $\mathcal{V}_{K}$ which has a regular model $Y$ over $S$. Let $\alpha \in C H^{p}\left(Y_{K} \times_{K} X_{K}\right)_{\mathbf{Q}}$ be a correspondence. For suitable $x, y$, we get from 4.1 the formula

$$
<x, \alpha_{C H}(y)>_{X_{K}}=<\left({ }^{t} \alpha\right)_{C H}(x), y>_{Y_{K}} .
$$

The Lefschetz operator $L: C H^{p}\left(X_{K}\right) \longrightarrow C H^{p+1}\left(X_{K}\right), \alpha \mapsto \alpha . c_{1}(\mathcal{L})$ associated with a line bundle $\mathcal{L}$ on $X_{K}$ induces a map on $C H\left(X_{K}\right)^{0}$. The height pairing should be non degenerate as a consequence of the following conjecture of Beilinson $([\mathrm{Be}]$, 5.3 and 5.5).

Conjecture 5.2. (Beilinson) Let $\mathcal{L}$ be ample and $2 p \leq d+1$. 
i) The operator

$$
L^{d+1-2 p}: C H^{p}\left(X_{K}\right)_{\mathbf{Q}}^{0} \longrightarrow C H^{d+1-p}\left(X_{K}\right)_{\mathbf{Q}}^{0}
$$

is an isomorphism.

ii) If $x \in C H^{p}\left(X_{K}\right)_{\mathbf{Q}}^{0}, x \neq 0$, and $L^{d+2-2 p}(x)=0$ then

$$
(-1)^{p}<x, L^{d+1-2 p} x>_{X_{K}}>0 .
$$

\section{The HeIGHT PAIRING ON CURVES}

Let $C_{K}$ be a curve of positive genus in $\mathcal{V}_{K}$ which is geometrically irreducible. After a finite extension of the ground field $K$, we may assume that $C_{K}$ has a regular semi-stable model $C$ over $S=\operatorname{Spec}\left(\mathcal{O}_{K}\right)$. This model satisfies

$$
C H^{1}\left(C_{K}\right)_{\mathbf{Q}}^{0}=C H^{1}\left(C_{K}\right)_{\mathbf{Q}}^{00} \text {. }
$$

This follows from $[\mathrm{Hr}]$, Th. 1.3, and is also shown in [Fa], Th. 4, [MB], 6.14.1. Hence we get a well defined pairing

$$
<.,>_{C_{K}}: C H^{1}\left(C_{K}\right)_{\mathbf{Q}}^{0} \times C H^{1}\left(C_{K}\right)_{\mathbf{Q}}^{0} \longrightarrow \mathbf{R} .
$$

It has been shown independently by Faltings and Hriljac that this pairing has a description in terms of the Néron-Tate pairing on the Jacobian of the curve. Let $\bar{K}$ be an algebraic closure of $K$ and $\operatorname{Jac}\left(C_{\bar{K}}\right)$ the Jacobian of $C_{\bar{K}}$. The latter carries a canonical polarization $\lambda_{C}: \operatorname{Jac}\left(C_{\bar{K}}\right) \longrightarrow \operatorname{Jac}\left(C_{\bar{K}}\right)^{\vee}([\mathrm{MB}], 2.6 .4,[\mathrm{Mi}], 6.11)$. We denote by $\theta^{1}$ the Abel-Jacobi map

$$
\theta^{1}: A^{1}\left(C_{K}\right) \longrightarrow \operatorname{Jac}\left(C_{\bar{K}}\right)(\bar{K})
$$

\section{Theorem 6.1. (Faltings, Hriljac)}

$$
\frac{1}{[K: \mathbf{Q}]}<x, y>_{C_{K}}=-\left(\theta^{1}(x), \lambda_{C} \circ \theta^{1}(y)\right)_{\operatorname{Jac}\left(C_{\overline{\mathbf{Q}}}\right)} \quad \text { for } \quad x, y \in A^{1}\left(C_{K}\right)
$$

Proof. [MB], Th. 6.15, [Fa], Th. 4, or [Hr] Th. 3.1.

Observe that the theorem describes (20) completely as we have

$$
A^{1}\left(C_{K}\right)_{\mathbf{Q}}=C H^{1}\left(C_{K}\right)_{\mathbf{Q}}^{0}
$$




\section{Higher Picard varieties}

In this section, we recall the theory of higher Picard varieties as developped by H. Saito building on ideas of Grothendieck and Liebermann. All the results in this section can be found in [S]. Therefore, we give no further references in the text. Let $k$ be an algebraically closed field and $X$ a variety in $\mathcal{V}_{k}$ of dimension $d$.

Definition 7.1. Let $A$ be an abelian variety over $k$ and

$$
h: A^{p}(X) \longrightarrow A(k)
$$

a group homomorphism.

i) $h$ is regular if for all varieties $Y$ in $\mathcal{V}_{k}$, all points $y_{0} \in Y(k)$, and all $\alpha \in$ $C H^{p}\left(Y \times_{k} X\right)$ the map

$$
Y(k) \longrightarrow A^{p}(X) \stackrel{h}{\longrightarrow} A(k), y \mapsto h\left(\alpha_{C H}\left([y]-\left[y_{0}\right]\right)\right)
$$

is induced from a morphism of varieties.

ii) $h$ is called a Picard homomorphism if there exists a variety $T$ in $\mathcal{V}_{k}$ and a correspondence $\alpha \in C H^{d+1-p}\left(X \times_{k} T\right)$ such that $A$ is a subvariety of the Picard variety $\mathrm{Pic}_{T / k}^{0}$ of $T$ and

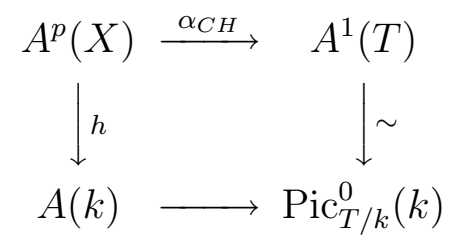

commutes.

Every Picard homomorphism is regular. It was shown by Saito that there exists an abelian variety $\operatorname{Pic}^{p}(X)$ and a universal Picard homomorphism

$$
\theta^{p}: A^{p}(X) \longrightarrow \operatorname{Pic}^{p}(X)(k)
$$

uniquely determined (up to unique isomorphism) by the property that for every Picard homomorphism $h: A^{p}(X) \longrightarrow A(k)$ there exists a unique homomorphism $f$ from $\operatorname{Pic}^{p}(X)$ to $A$ such that $h=f \circ \theta^{p}$. The abelian variety $\operatorname{Pic}^{p}(X)$ is called the $p$-th Picard variety of $X$.

Let us recall some properties of $\operatorname{Pic}^{p}(X)$. The abelian varieties $\operatorname{Pic}^{1}(X)$ and $\operatorname{Pic}^{d}(X)$ are the Picard and the Albanese variety of $X$. We consider the subgroup $I^{p}(X)$ of $A^{p}(X)$ consisting of elements $u \in A^{p}(X)$ which satisfy $\alpha_{C H}(u)=0$ in $C H^{1}(T)$ for every variety $T$ in $\mathcal{V}_{k}$ and every correspondence $\alpha \in C H^{d-p+1}\left(X \times_{k} T\right)$. This is the subgroup of cycles incidence equivalent to zero. The map $\theta^{p}$ induces an isomorphism $A^{p}(X) / I^{p}(X) \stackrel{\sim}{\longrightarrow} \operatorname{Pic}^{p}(X)(k)$. Let $Y$ be a variety in $\mathcal{V}_{k}$ of dimension $e$. For every correspondence $\alpha \in C H^{e-p+q}\left(Y \times_{k} X\right)$, there is one and only one 
homomorphism $\operatorname{Pic}(\alpha): \operatorname{Pic}^{p}(Y) \longrightarrow \operatorname{Pic}^{q}(X)$ such that the diagram

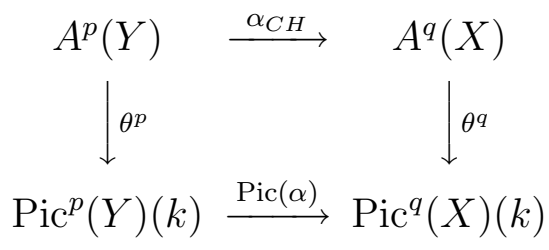

commutes. This yields a canonical map $C H^{p}\left(Y \times_{k} X\right) \longrightarrow \operatorname{Hom}\left(\operatorname{Alb}(Y), \operatorname{Pic}^{p}(X)\right)$ for every $Y$ in $\mathcal{V}_{k}$. For $Y=\operatorname{Pic}^{p}(X)$, it becomes

$$
\beta: C H^{p}\left(\operatorname{Pic}^{p}(X) \times_{k} X\right) \longrightarrow \operatorname{End}\left(\operatorname{Pic}^{p}(X)\right) .
$$

The intersection $\operatorname{Im}(\beta) \cap \mathbf{Z} \cdot \operatorname{id}_{\operatorname{Pic}^{p}(X)}$ can be shown to be equal to $\left(k_{X}^{p} \mathbf{Z}\right) \cdot \operatorname{id}_{\operatorname{Pic}^{p}(X)}$ for some positive integer $k_{X}^{p}$. An element $\alpha_{X}^{p}$ in $C H^{p}\left(\operatorname{Pic}^{p}(X) \times_{k} X\right)$ that is mapped under $\beta$ to $k_{X}^{p} \cdot \operatorname{id}_{\mathrm{Pic}^{p}(X)}$, is called a Poincaré $p$-cycle. Let $\alpha_{X}^{p}$ be a Poincaré $p$-cycle. The induced map

$$
\operatorname{Pic}^{p}(X)=\operatorname{Alb}\left(\operatorname{Pic}^{p}(X)\right) \stackrel{\operatorname{Pic}\left(\alpha_{X}^{p}\right)}{\longrightarrow} \operatorname{Pic}^{p}(X)
$$

is by definition multiplication by $k_{X}^{p}$ on the abelian variety $\operatorname{Pic}^{p}(X)$. The transposed correspondence ${ }^{t}\left(\alpha_{X}^{p}\right) \in C H^{p}\left(X \times \operatorname{Pic}^{p}(X)\right)$ defines the duality homomorphism

$$
\lambda_{X}^{d+1-p}=\operatorname{Pic}\left({ }^{t} \alpha_{X}^{p}\right): \operatorname{Pic}^{d+1-p}(X) \longrightarrow \operatorname{Pic}^{p}(X)^{\vee}=\operatorname{Pic}^{1}\left(\operatorname{Pic}^{p}(X)\right) .
$$

The map $\lambda_{X}^{p}$ is an isogeny which is independent of the choice of the Poincaré $p$-cycle $\alpha_{X}^{p}$. Given a correspondence $\alpha \in C H^{p+q}(Y \times X)$, one has

$$
k_{X}^{p} \lambda_{Y}^{q+1} \circ \operatorname{Pic}\left({ }^{t} \alpha\right)=k_{Y}^{e-q} \operatorname{Pic}(\alpha)^{\vee} \circ \lambda_{X}^{d+1-p} .
$$

For a curve $C$, we have $k_{C}^{1}=1$ and $\lambda_{C}^{1}=\operatorname{mult}(-1) \circ \lambda_{C}$ where $\lambda_{C}$ is the canonical polarization of the Jacobian of $C$. The second equality can be derived from [Mi], 6.11 .

\section{Height Pairing BetWeen Algebraically trivial CyCles}

The height pairing between cycles algebraically equivalent to zero has a description in terms of the Néron-Tate pairing on the higher Picard varieties.

Let $X_{K}$ be a variety in $\mathcal{V}_{K}$ which has a regular model $X$ over $S$.

Lemma 8.1. $\quad A^{p}\left(X_{K}\right)_{\mathbf{Q}} \subset C H^{p}\left(X_{K}\right)_{\mathbf{Q}}^{00}$ for all $p$.

Proof. Let $x$ be a class in $A^{p}\left(X_{K}\right)$. Merely by definition, there exist a finite extension $L$ of $K$, a geometrically irreducible curve $C_{L}$ defined over $L$, an element $z \in A^{1}\left(C_{L}\right)$ and a correspondence $\alpha \in C H^{p}\left(C_{L} \times_{L} X_{L}\right)$ such that $\alpha_{C H}(z)$ equals $x_{L}$, the image of $x$ in $A^{p}\left(X_{L}\right)([\mathrm{Fu}], 10.3 .2)$. We may also assume that $C_{L}$ has a regular semistable model $C$ over $S^{\prime}=\operatorname{Spec}\left(\mathcal{O}_{L}\right)$.

According to (19), we may write $z=\xi\left(z^{\prime}\right)$ for some $z^{\prime} \in C H_{\text {fin }}^{1}(C)_{\mathbf{Q}}^{\perp}$. Let $\alpha^{\prime}$ be a correspondence in $C H^{p}\left(C \times_{S^{\prime}} X_{S^{\prime}}\right)$ which restricts to $\alpha$. The element $x_{L}^{\prime}=\alpha_{C H}^{\prime}\left(z^{\prime}\right)$ is a preimage of $x_{L}$ in $C H^{p}\left(X_{S^{\prime}}\right)_{\mathbf{Q}}$. We consider $x^{\prime}=[L: K]^{-1} p_{*}\left(x_{L}^{\prime}\right)$ where 
$p: X_{S^{\prime}} \rightarrow X$. This element satisfies $\xi\left(x^{\prime}\right)=x$. It remains to show that it is contained in $C H_{\text {fin }}^{d+1-p}(X)_{\mathbf{Q}}^{\perp}$. Given $y^{\prime} \in C H_{\text {fin }}^{d+1-p}(X)_{\mathbf{Q}}$, we have

$$
\begin{array}{rlr}
{[L: K]<y^{\prime}, x^{\prime}>_{X}} & =<y^{\prime}, p_{*} \alpha_{C H}^{\prime}\left(z^{\prime}\right)>_{X} & \\
& =<y^{\prime}, \alpha_{C H}^{\prime}\left(z^{\prime}\right)>_{p} & (\text { by }(7)) \\
& =<z^{\prime},\left({ }^{t} \alpha^{\prime}\right)_{C H}\left(y^{\prime}\right)>_{C} & (\text { by } 4.1) \\
& =0
\end{array}
$$

as $\left({ }^{t} \alpha^{\prime}\right)_{C H}\left(y^{\prime}\right)$ is easily seen to be an element of $C H_{\text {fin }}^{1}(C)_{\mathbf{Q}}$.

Hence there is a well defined pairing $A^{p}\left(X_{K}\right) \times A^{d+1-p}\left(X_{K}\right) \longrightarrow \mathbf{R}$ which has the following description.

Theorem 8.2. Given $x \in A^{p}\left(X_{K}\right)$ and $y \in A^{d+1-p}\left(X_{K}\right)$, we have

$$
\frac{1}{[K: \mathbf{Q}]}<x, y>_{X_{K}}=\frac{1}{k_{X}^{p}}\left(\theta^{p}(x), \lambda_{X}^{d+1-p} \circ \theta^{d+1-p}(y)\right)_{\operatorname{Pic}^{p}\left(X_{\bar{K}}\right)} .
$$

Proof. We choose $x^{\prime}=[L: K]^{-1} p_{*} \alpha_{C H}^{\prime}\left(z^{\prime}\right)$ as in the proof of 8.1. Let $y^{\prime}$ be a lift of $y$ to $C H_{\text {fin }}^{p}(X)_{\mathbf{Q}}^{\perp}$. As in the proof of 8.1, we get

$$
\begin{aligned}
{[K: \mathbf{Q}]^{-1}<x, y>_{X_{K}} } & =[L: \mathbf{Q}]^{-1}<y^{\prime}, \alpha_{C H}^{\prime}\left(z^{\prime}\right)>_{p} \\
& =[L: \mathbf{Q}]^{-1}<z^{\prime},\left({ }^{t} \alpha^{\prime}\right)_{C H}\left(y^{\prime}\right)>_{C} \\
& =-\left(\theta^{1}(z), \lambda_{C} \circ \theta^{1} \circ\left({ }^{t} \alpha\right)_{C H}(y)\right)_{\operatorname{Jac}\left(C_{\bar{K}}\right)} \\
& =\left(\theta^{1}(z), \lambda_{C}^{1} \circ \operatorname{Pic}\left({ }^{t} \alpha\right) \circ \theta^{d+1-p}(y)\right)_{\operatorname{Jac}\left(C_{\bar{K}}\right)} \\
& =\left(\theta^{1}(z), \frac{k_{C}^{1}}{k_{X}^{p}} \operatorname{Pic}(\alpha)^{\vee} \circ \lambda_{X}^{d+1-p} \circ \theta^{d+1-p}(y)\right)_{J_{\operatorname{Jac}\left(C_{\bar{K}}\right)}} \\
& =\left(k_{X}^{p}\right)^{-1}\left(\operatorname{Pic}(\alpha) \circ \theta^{1}(z), \lambda_{X}^{d+1-p} \circ \theta^{d+1-p}(y)\right)_{\operatorname{Pic}^{p}\left(X_{\bar{K}}\right)} \\
& =\left(k_{X}^{p}\right)^{-1}\left(\theta^{p}(x), \lambda_{X}^{d+1-p} \circ \theta^{d+1-p}(y)\right)_{\operatorname{Pic}^{p}\left(X_{\bar{K}}\right) .}
\end{aligned}
$$

This proves our theorem.

\section{A polarization of the Higher PicARD VARIETy of AN ABELIAN VARIETy}

We consider a $g$-dimensional abelian variety $A$ defined over a field $k$. Let $\lambda: A \rightarrow$ $A^{\vee}$ be a polarization of $A$. We choose a Poincaré bundle $\mathcal{P}$ on $A \times_{k} A^{\vee}$ and define

$$
D_{\lambda}^{p}=(-1)^{g+p}\left(\mathrm{id} \times_{k} \lambda\right)^{*} c_{1}(\mathcal{P})^{2 g+1-2 p} \in C H^{2 g+1-2 p}\left(A \times_{k} A\right) .
$$

In the following, we assume that $k$ is an algebraically closed field of characteristic zero. The correspondence $D_{\lambda}^{p}$ induces a morphism

$$
\Lambda_{\lambda}^{p}: \operatorname{Pic}^{p}(A) \stackrel{\operatorname{Pic}\left(D_{\lambda}^{p}\right)}{\longrightarrow} \operatorname{Pic}^{g+1-p}(A) \stackrel{\lambda_{A}^{g+1-p}}{\longrightarrow} \operatorname{Pic}^{p}(A)^{\vee} .
$$


Theorem 9.1. The morphism $\Lambda_{\lambda}^{p}$ defines a polarization of $\operatorname{Pic}^{p}(A)$.

We are going to derive this result in section 11 from the corresponding statement for Weil's intermediate Jacobian.

\section{Relation With intermediate Jacobians}

We briefly recall some facts about Weil's intermediate Jacobians. Let $X$ be a projective complex manifold of dimension $d$. The endomorphism $C$ of $H^{\bullet}(X, \mathbf{C})$ is defined in terms of the Hodge decomposition $H^{\bullet}(X, \mathbf{C})=\oplus_{p, q} H^{p, q}(X)$. It acts on $H^{p, q}(X)$ by multiplication with $\sqrt{-1}^{p-q}$. Furthermore, it induces a map on $H^{\bullet}(X, \mathbf{R})$ where it defines the structure of a complex vector space via

$$
(a+\sqrt{-1} b) \alpha=a \alpha-b C(\alpha), a, b \in \mathbf{R}, \alpha \in H^{\bullet}(X, \mathbf{R}) .
$$

The image of $H^{\bullet}(X, \mathbf{Z})$ in $H^{\bullet}(X, \mathbf{R})$ is a lattice which we denote by $H^{\bullet}(X)_{\mathbf{z}}$. The $p$-th intermediate Jacobian $J^{p}(X)$ of $X$ in the sense of Weil is by definition the complex torus

$$
J^{p}(X)=H^{2 p-1}(X, \mathbf{R}) / H^{2 p-1}(X) \mathbf{z}
$$

The complex tori $J^{p}(X)$ and $J^{d+1-p}(X)$ are dual to each other in the following sense. The pairing

$$
q: H^{\bullet}(X, \mathbf{R}) \times H^{\bullet}(X, \mathbf{R}) \longrightarrow \mathbf{C}, q(\alpha, \beta)=\int_{X}(\sqrt{-1} \alpha) \wedge \beta+\sqrt{-1} \int_{X} \alpha \wedge \beta
$$

is a non degenerate hermitian form on $H^{\bullet}(X, \mathbf{R})$. It induces an isomorphism

$$
\text { pd : } H^{2 d+1-2 p}(X, \mathbf{R}) \longrightarrow H^{2 p-1}(X, \mathbf{R})^{\vee}=\operatorname{Hom}_{\mathbf{C}-\text { antilinear }}\left(H^{2 p-1}(X, \mathbf{R}), \mathbf{C}\right)
$$

which identifies the image of $H^{2 d+1-2 p}(X)_{\mathbf{z}}$ with the dual lattice $\left(H^{2 p-1}(X)_{\mathbf{z}}\right)^{\vee}$. This yields a natural identification

$$
\mathrm{pd}: J^{d+1-p}(X) \longrightarrow J^{p}(X)^{\vee} .
$$

A correspondence $\alpha \in H^{\bullet}(X \times Y, \mathbf{Z})$ with $C \alpha=\alpha$ defines in a standard manner maps $H(\alpha): H^{\bullet}(X, \mathbf{R}) \rightarrow H^{\bullet}(Y, \mathbf{R})$ and $J(\alpha): J^{\bullet}(X) \rightarrow J^{\bullet}(Y)$. The intermediate Jacobian comes with an Abel-Jacobi map

$$
\Phi^{p}: C H^{p}(X)^{0} \longrightarrow J^{p}(X)(\mathbf{C})
$$

which is defined and discussed in [L]. A cycle is called Abel-Jacobi equivalent to zero if it is contained in the kernel of $\Phi^{p}$. The image of the group $A^{p}(X)$ of cycles algebraically equivalent to zero is denoted by $J_{a}^{p}(X)$. It can be shown that $J_{a}^{p}(X)$ is an abelian subvariety of $J^{p}(X)$. There is a canonical homomorphism

$$
h^{p}: J_{a}^{p}(X) \longrightarrow \operatorname{Pic}^{p}(X)
$$

which satisfies $h^{p} \circ \Phi^{p}=\theta^{p}$ ([S], 5.1.2). The morphism $h^{p}$ is surjective. It is an isogeny if $X$ is an abelian variety ([S], 5.2). This holds in fact more generally if numerical and homological equivalence on $X$ agree up to torsion ([Kl]). 


\section{Proof of Theorem 9.1}

Let $k$ be an algebraically closed field which admits an embedding $\sigma: k \hookrightarrow \mathbf{C}$ into the complex numbers. Let $X$ be a variety in $\mathcal{V}_{k}$. We are going to relate $\operatorname{Pic}^{p}(X), \operatorname{Pic}^{p}\left(X_{\mathbf{C}}\right)$, and $J_{a}^{p}\left(X_{\mathbf{C}}\right)$. The map $\theta^{p}: A^{p}(X) \longrightarrow \operatorname{Pic}^{p}(X)(k)$ is a Picard homomorphism. Hence we can find a variety $T$ and a correspondence $\alpha$ for $\theta^{p}$ as in 7.1. We choose a Poincaré cycle $\alpha_{X}^{p}$ and obtain a commutative diagram

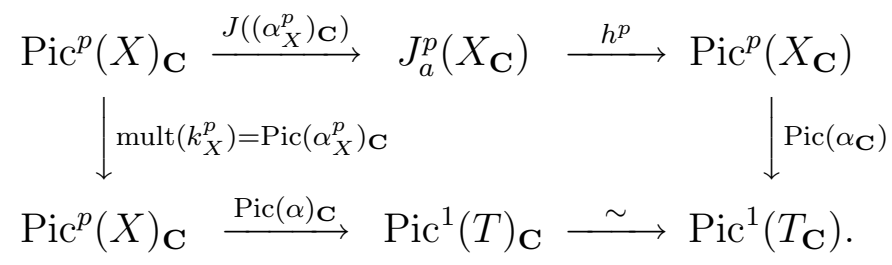

We conclude that the map

$$
j_{X}=J\left(\left(\alpha_{X}^{p}\right)_{\mathbf{C}}\right): \operatorname{Pic}^{p}(X)_{\mathbf{C}} \longrightarrow J^{p}\left(X_{\mathbf{C}}\right)
$$

has a finite kernel contained in the $k_{X}^{p}$-torsion.

We consider again an abelian variety $A$ with polarization $\lambda$ defined over an algebraically closed field $k$ of characteristic zero. In order to prove 9.1, we may assume without loss of generality that $k$ admits an embedding $\sigma: k \hookrightarrow \mathbf{C}$. We denote the dimension of $A$ by $g$ and the degree of $\lambda$ by $v^{2}$. Theorem 9.1 will be a consequence of the following lemmata. The first reproduces more or less Weil's proof that his intermediate Jacobians are abelian varieties.

Lemma 11.1. The morphism

$$
\widetilde{\Lambda_{\lambda}^{p}}: J^{p}\left(A_{\mathbf{C}}\right) \stackrel{J\left(D_{\lambda}^{p}\right)}{\longrightarrow} J^{g+1-p}\left(A_{\mathbf{C}}\right) \stackrel{\mathrm{pd}}{\longrightarrow} J^{p}\left(A_{\mathbf{C}}\right)^{\vee}
$$

defines a polarization of $J^{p}\left(A_{\mathbf{C}}\right)$.

Proof. The morphism $\widetilde{\Lambda_{\lambda}^{p}}$ is a polarization if it is an isogeny and the induced representation $H\left(D_{\lambda}^{p}\right): H^{2 p-1}\left(A_{\mathbf{C}}, \mathbf{R}\right) \rightarrow H^{2 g+1-2 p}\left(A_{\mathbf{C}}, \mathbf{R}\right)$ defines a positive definite hermitian form $(\alpha, \beta) \mapsto q\left(H\left(D_{\lambda}^{p}\right)(\alpha), \beta\right)$ on $H^{2 p-1}\left(A_{\mathbf{C}}, \mathbf{R}\right)$. Let '*' be the Hodge*-operator on the complex manifold $A_{\mathbf{C}}$ associated with the polarization of $A$ given by $\lambda$. According to $[\mathrm{Ku}]$, Prop. 6.4, we have

$$
H\left(D_{\lambda}^{p}\right)(\alpha)=-v(2 g+1-2 p) \text { ! } * C(\alpha) \text { for } \alpha \in H^{2 p-1}\left(A_{\mathbf{C}}, \mathbf{R}\right) .
$$

Hence, $\widetilde{\Lambda_{\lambda}^{p}}$ is an isogeny as ' $*$ ' induces an isomorphism on the tangent spaces. The positivity of the Hodge-*-operator implies immediately that the hermitian form $q\left(H\left(D_{\lambda}^{p}\right)(),.\right)$ is positive definite.

Lemma 11.2. Let $A, B$ be abelian varieties over $k$.

i) If $\lambda: A \rightarrow A^{\vee}$ is a morphism, $n$ a positive integer, and $n \lambda$ a polarization then $\lambda$ is a polarization.

ii) Let $f: A \longrightarrow B$ be a homomorphism with finite kernel and $\lambda_{B}: B \rightarrow B^{\vee} a$ polarization of $B$. Then $\lambda_{A}=f^{\vee} \circ \lambda_{B} \circ f$ is a polarization of $A$. 
iii) Let $\lambda: A \rightarrow A^{\vee}$ be a homomorphism such that $\lambda_{\mathbf{C}}$ is a polarization of $A_{\mathbf{C}}$. Then $\lambda$ is a polarization of $A$.

Proof. i) A polarization of $A$ is a morphism

$$
\Lambda(\mathcal{L}): A \longrightarrow A^{\vee}, a \mapsto t_{a}^{*} \mathcal{L} \otimes \mathcal{L}^{-1}
$$

for some ample line bundle $\mathcal{L}$ on $A$. Assume that $n \lambda=\Lambda(\mathcal{L})$ for some ample line bundle $\mathcal{L}$. It follows from $[\mathrm{Mu}], \S 23$. Th. 3 , that $\mathcal{L} \cong \mathcal{M}^{\otimes n}$ for some line bundle $\mathcal{M}$. Therefore $\lambda=\Lambda(\mathcal{M})$ is a polarization. To prove ii), we suppose $\lambda_{B}=\Lambda(\mathcal{L})$ for some ample line bundle $\mathcal{L}$ on $B$. One easily gets $\lambda_{A}=\Lambda\left(f^{*} \mathcal{L}\right)$. This implies our claim as $f^{*} \mathcal{L}$ is still ample ([Ha], III Ex. 5.7). It remains to show iii). We have $2 \lambda_{\mathbf{C}}=\Lambda\left(L^{\Delta}(\lambda)_{\mathbf{C}}\right)$ where $L^{\Delta}(\lambda)=(\mathrm{id}, \lambda)^{*} \mathcal{P}$ and $\mathcal{P}$ is the Poincaré bundle on $A \times_{k} A^{\vee}$. The line bundle $L^{\Delta}(\lambda)_{\mathbf{C}}$ is ample as $\lambda_{\mathbf{C}}$ is a polarization. The cohomological criterion for ampleness shows the same is true for $L^{\Delta}(\lambda)$. Hence $2 \lambda=\Lambda\left(L^{\Delta}(\lambda)\right)$ is a polarization.

Proof of Theorem 9.1. Our first observation is the equality

$$
\left(\Lambda_{\lambda}^{p}\right)_{\mathbf{C}} \circ \operatorname{mult}\left(k_{A}^{p}\right)=j_{A}^{\vee} \circ \widetilde{\Lambda_{\lambda}^{p}} \circ j_{A}
$$

in $\operatorname{End}\left(\operatorname{Pic}^{p}(A)_{\mathbf{C}}\right)$. It holds if we show that the induced maps on the tangent spaces agree. This follows from the diagram ( $d$ denotes the dimension of $\left.\operatorname{Pic}^{p}(A)\right)$

$$
\begin{aligned}
& H^{2 d-1}\left(\operatorname{Pic}^{p}(A)_{\mathbf{C}}, \mathbf{R}\right) \stackrel{H\left({ }^{t} \alpha_{A}^{p} \circ D_{\lambda}^{p} \circ \alpha_{A}^{p}\right)}{\longrightarrow} H^{1}\left(\operatorname{Pic}^{p}(A)_{\mathbf{C}}, \mathbf{R}\right) \stackrel{\mathrm{pd}}{\longrightarrow} H^{2 d-1}\left(\operatorname{Pic}^{p}(A)_{\mathbf{C}}, \mathbf{R}\right)^{\vee} \\
& \downarrow H\left(\alpha_{A}^{p}\right) \quad \uparrow H\left({ }^{t} \alpha_{A}^{p}\right) \quad \uparrow H\left(\alpha_{A}^{p}\right)^{\vee} \\
& H^{2 p-1}\left(A_{\mathbf{C}}, \mathbf{R}\right) \quad \stackrel{H\left(D_{\lambda}^{p}\right)}{\longrightarrow} \quad H^{2 g+1-2 p}\left(A_{\mathbf{C}}, \mathbf{R}\right) \stackrel{\mathrm{pd}}{\longrightarrow} \quad H^{2 p-1}\left(A_{\mathbf{C}}, \mathbf{R}\right)^{\vee}
\end{aligned}
$$

which is easily seen to commute.

An application of 11.1 and 11.2 to (26) yields our claim.

\section{Applichtion: A Hodge index theOREM FOR AlgEBRAicAlly tRivial CYCLES MODULO INCIDENCE EQUIVALENCE}

We investigate the subquotient of $C H^{p}\left(X_{K}\right)_{\mathbf{Q}}^{0}$ given by cycles algebraically equivalent to zero modulo incidence equivalence. Let $\bar{K}$ be an algebraic closure of $K$. The group $I^{p}\left(X_{\bar{K}}\right)$ of cycle classes incidence equivalent to zero on $X_{\bar{K}}$ was defined in section 7 . We define $I^{p}\left(X_{K}\right)=\operatorname{Ker}\left(A^{p}\left(X_{K}\right) \longrightarrow A^{p}\left(X_{\bar{K}}\right) / I^{p}\left(X_{\bar{K}}\right)\right)$ and

$$
B^{p}\left(X_{K}\right)=A^{p}\left(X_{K}\right) / I^{p}\left(X_{K}\right)
$$

Let $A_{K}$ be an abelian variety of dimension $g$ defined over $K$ which has a regular model over $S=\operatorname{Spec}\left(\mathcal{O}_{K}\right)$. The height pairing induces a pairing

$$
<.,>_{A_{K}}: B^{p}\left(A_{K}\right)_{\mathbf{Q}} \times B^{g+1-p}\left(A_{K}\right)_{\mathbf{Q}} \longrightarrow \mathbf{R} .
$$


We fix an embedding of $\bar{K}$ into $\mathbf{C}$. Observe that our considerations in section 11 show that $B^{p}\left(A_{K}\right)_{\mathbf{Q}}$ is the quotient of $A^{p}\left(A_{K}\right)_{\mathbf{Q}}$ by the subspace of cycles which are Abel-Jacobi equivalent to zero. Beilinson and Bloch conjecture ([Be], 5.6)

$$
B^{p}\left(A_{K}\right)_{\mathbf{Q}}=A^{p}\left(A_{K}\right)_{\mathbf{Q}} .
$$

Part i) of the following theorem was shown by Lieberman over the complex numbers $([\mathrm{L}], 7.2)$.

Theorem 12.1. Let $\mathcal{L}$ be an ample line bundle on $A_{K}$ and $2 p \leq g+1$.

i) The operator

is an isomorphism.

$$
L^{g+1-2 p}: B^{p}\left(A_{K}\right)_{\mathbf{Q}} \longrightarrow B^{g+1-p}\left(A_{K}\right)_{\mathbf{Q}}
$$

ii) If $x \in B^{p}\left(A_{K}\right)_{\mathbf{Q}}, x \neq 0$, and $L^{g+2-2 p}(x)=0$ then

$$
(-1)^{p}<x, L^{g+1-2 p} x>_{A_{K}}>0 .
$$

Proof. Our first observation is

$$
\left.\operatorname{mult}(n)^{*}\right|_{H^{2 p-1}\left(A_{\mathbf{C}}, \mathbf{R}\right)}=n^{2 p-1} \text { id for } n \in \mathbf{Z} \text {. }
$$

This yields the same formula for $\operatorname{mult}(n)^{*}$ on $J^{p}\left(A_{\mathbf{C}}\right)$ instead of $H^{2 p-1}\left(A_{\mathbf{C}}, \mathbf{R}\right)$. The inclusions

$$
B^{p}\left(A_{K}\right)_{\mathbf{Q}} \subset\left(\operatorname{Pic}^{p}\left(A_{\bar{K}}\right)(\bar{K})\right)_{\mathbf{Q}} \stackrel{j_{A_{A}}}{\hookrightarrow}\left(J^{p}\left(A_{\mathbf{C}}\right)(\mathbf{C})\right)_{\mathbf{Q}}
$$

are compatible with $n$-multiplication. Hence, we get

$$
\left.\operatorname{mult}(n)^{*}\right|_{B^{p}\left(A_{K}\right)_{\mathbf{Q}}}=n^{2 p-1} \text { id for } n \in \mathbf{Z} \text {. }
$$

As a consequence, we see that the Lefschetz operator associated with $\mathcal{L}^{\otimes 2}$ acts like the Lefschetz operator associated with $\mathcal{L} \otimes \operatorname{mult}(-1)^{*} \mathcal{L}$ on $B^{p}\left(A_{K}\right)_{\mathbf{Q}}$. Therefore, we may assume that $\mathcal{L}$ is symmetric. Under this assumption, statement i) follows from $[\mathrm{Ku}]$, Th. 5.2 as the functors $B^{p}(.)_{\mathbf{Q}}$ factor through Chow motives.

We will need Theorem 9.1 in order to show ii). The positivity of the Néron-Tate height implies in combination with Theorem 8.2.

$$
\frac{1}{[K: \mathbf{Q}]}<x,\left(D_{\lambda}^{p}\right)_{C H}(x)>_{A_{K}}=\frac{1}{k_{A}^{p}}\left(\theta^{p}(x), \Lambda_{\lambda}^{p} \circ \theta^{p}(x)\right)_{\operatorname{Pic}^{p}\left(A_{\bar{K}}\right)}>0
$$

for all non zero $x \in B^{p}\left(A_{K}\right)_{\mathbf{Q}}$. This implies our claim as we have

$$
L^{d+1-2 p}(x)=(-1)^{p} \frac{(g+1-2 p) !}{v(2 g+1-2 p) !}\left(D_{\lambda}^{p}\right)_{C H}(x)
$$

for every $x \in B^{p}\left(X_{K}\right)_{\mathbf{Q}}$ which satisfies $L^{d+2-2 p}=0$ by $[\mathrm{Ku}]$, Th. 6.2.

Remark 12.2. Using nearly the same arguments as above, we can prove the last theorem for an arbitrary variety $X_{K}$ in $\mathcal{V}_{K}$ under the following assumptions:

i) Grothendieck's conjecture of Lefschetz type in [Gr] holds for $X_{\bar{K}}$,

ii) the intersection product in $C H^{\bullet}\left(X_{K}\right)_{\mathbf{Q}}^{0}$ is zero ([Be], Conj. 5.7), 
iii) the Künneth components $\pi_{i}$ induce the zero map on $B^{p}\left(X_{K}\right)_{\mathbf{Q}}$ for all $i \neq$ $2 p-1$.

The first assumption implies that the Künneth components $\pi_{i}$ of the diagonal are algebraic. The $\pi_{i}$ are invariant under the action of the absolute Galois group of $K$. They are idempotents in the ring of correspondences from $X_{K}$ to itself formed by cycles modulo homological equivalence with rational coefficients. Assumption ii) assures that the $\pi_{i}$ act on $C H^{\bullet}\left(X_{K}\right)_{\mathbf{Q}}^{0}$ and $B^{\bullet}\left(X_{K}\right)_{\mathbf{Q}}$.

Remark. After writing this paper, I learned from unpublished notes of J. Murre that he also has related the height pairing to Saito's higher Picard varieties. In his notes, dated from 1986, Murre uses the righthand side of (23) to define a height pairing between cycles algebraically equivalent to zero.

Acknowledgements. I am grateful to E. Bachmat and A. Werner for their comments on a first version of this paper.

\section{REFERENCES}

[Be] Beilinson, A.A.: Height pairings between algebraic cycles. In: Yu. I. Manin: K-theory, Arithmetic and Geometry, Moscow 1984-86 (Lect. Notes Math., vol. 1289, pp. 1-25) Springer: Berlin-Heidelberg-New York 1987 and Contemp. Math. 67, 1-24 (1987)

[Bl] Bloch, S.: Height pairings for algebraic cycles. J. Pure Appl. Algebra 34, 119-145 (1984)

[Fa] Faltings, G.: Calculus on an arithmetic surface. Ann. of Math. 119, 387-424 (1984)

[Fu] Fulton, W.: Intersection theory. Springer: Berlin-Heidelberg-New York 1984

[GS1] Gillet, H., Soulé C.: Arithmetic intersection theory. Publ. Math. I.H.E.S. 72, 94-174 (1990)

[GS2] Gillet, H., Soulé C.: An arithmetic Riemann-Roch theorem. Inv. math. 110, 473-543 (1992)

[Gr] Grothendieck, A.: Standard conjectures on algebraic cycles. In: S. S. Abhyankhar et al.: Algebraic Geometry, Bombay 1968 (pp. 193-199) Oxford University Press: Oxford 1969

[Ha] Hartshorne, R.: Algebraic geometry. Springer: Berlin-Heidelberg-New York 1977

[Hr] Hriljac, P.: Heights and Arakelov's intersection theory. Amer. J. Math. 107, 23-38 (1985)

[Kl] Kleiman, S.: Finiteness theorems for algebraic cycles. Actes Congres Intern. Math. Nice, 1970, Tome 1, 445-450

[Ku] Künnemann, K. : A Lefschetz decomposition for Chow motives of abelian schemes. Invent. math. 113, 85-102 (1993)

[L] Lieberman, D. I.: Higher Picard varieties. Amer. J. Math. 90, 1165-1199 (1968)

[Mi] Milne, J.: Jacobian varieties. In: G. Cornell, J.H. Silverman, Arithmetic geometry, Storrs, 1984, Springer: Berlin-Heidelberg-New York 1986

[MB] Moret-Bailly, L.: Metriques permises. In: L. Szpiro: Séminaire sur les pinceaux arithmétiques: la conjecture de Mordell, Astérisque 127, 29-87 (1985)

$[\mathrm{Mu}]$ Mumford, D.: Abelian varieties. Oxford university press: Oxford 1970

[Né] Néron, A.: Quasi-fonctions et hauteurs sur les variétés abéliennes. Ann. of Math. 82, 249-331 (1965)

[S] Saito, H.: Abelian varieties attached to cycles of intermediate dimension. Nagoya Math. J. 75, 95-119 (1979)

Mathematisches Institut, Einsteinstrasse 62, 48149 Münster, Germany

E-mail address: kuenne@math.uni-muenster.de 